\title{
PlanNing Trajectory of THE Mobile Robot WITH A CAMERA
}

\author{
Alexey Katsurin \\ Institute of Automation and Control Processes, Far Eastern Federal University, Vladivostok, 690000 Russia
}

\begin{abstract}
The paper solved the task of planning trajectory of the car-like mobile robot using a movable camera installed on it. Using a developed system and algorithm of telecontrol the robot moves in the specified direction with any initial orientation of a movable camera and basis of robot. Proposed system of telecontrol by the mobile robot and algorithm of its work allows automatically to consider mutual orientation of a movable camera and the basis of the mobile robot. Such algorithm was developed and simulated on model.
\end{abstract}

Keyword: planning trajectory; mobile robot; telecontrol; movable camera; mechatronics
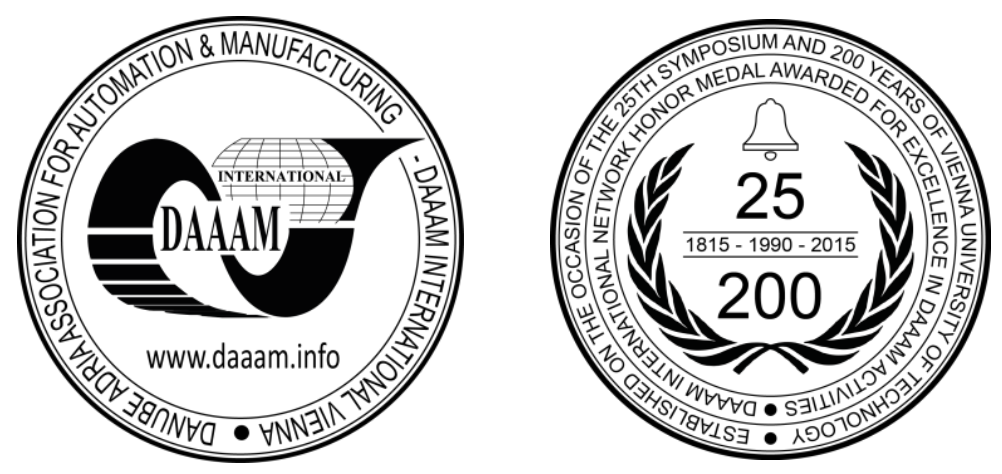

This Publication has to be referred as: Katsurin, A[lexey] (2016). Planning Trajectory of the Mobile Robot with a Camera, Proceedings of the 26th DAAAM International Symposium, pp.0407-0416, B. Katalinic (Ed.), Published by DAAAM International, ISBN 978-3-902734-07-5, ISSN 1726-9679, Vienna, Austria

DOI:10.2507/26th.daaam.proceedings.055 


\section{Introduction}

Now the mobile robots and manipulators with telecontrol are used for performance of various tasks in extreme and dangerous conditions, including search and prospecting works, neutralisations of ammunition, etc. [1-5]. Thus the operator on the basis of the image of working space on the screen of the monitor forms demanded control commands using the special setting device. The accurate, fast and qualitative decision of tasks is one of the basic requirements to the operator of the mobile robot. The big psychological load on the operator is the basic problem arising at telecontrol by the mobile robot, equipped with a mobile camera. He should consider constantly current spatial orientation, both the robot bases, and a camera during the setting of control commands.

The objective of the developed system remote control has been resolved for a mobile robot on a moving platform with four independently rotating wheels (differentially driven mobile robot) and mounted on the chassis platform rotating camera. However, the resulting solution is not applicable to mobile robots built by the type of vehicles consisting of two steering wheels in front and two rear driving wheels, as a result of the kinematic constraints - such a platform is not able to turn on a dime.

To solve the problem - related commands motion control of the mobile robot with the vector orientation view camera mounted on the unit. To achieve it, the trajectory, moving on a platform which is turning at a predetermined angle of orientation, thus combined the vector orientation of the platform and the camera view at the end point of the trajectory.

Thus the problem of development of new methods and algorithms of effective telecontrol by mobile robots with change of orientation of an optical axis of a camera remains still actual.

\section{Task setting}

The task of development of system of telecontrol by the mobile robot and algorithm of its work is setting and solving in paper for the decision of the specified problem. Algorithm allows considering automatically mutual orientation of a camera and the basis of the mobile robot. The operator sets control commands using image on the monitor. The robot moves in the specified direction at any initial orientation of a camera and the robot basis. Such algorithm has been developed, simulated on model and realised as the program module.

\section{The mathematical description of the trajectory}

Considered problem has two solutions: first solution - a platform begins to move along the path toward the original view of the camera, as the motion platform rotates as long as the angle of orientation the platform does not match with the initial orientation angle of view camera, this occurs at the end point formed by the trajectory on the motion is a straight line (graphic representation of this solution is shown in Figure 1), the second decision - the platform moves along a trajectory that takes the orientation of the platform to the desired angle at the end point of the trajectory, which is located at the point of reference (a graphic representation of the solutions presented in Figure 2).

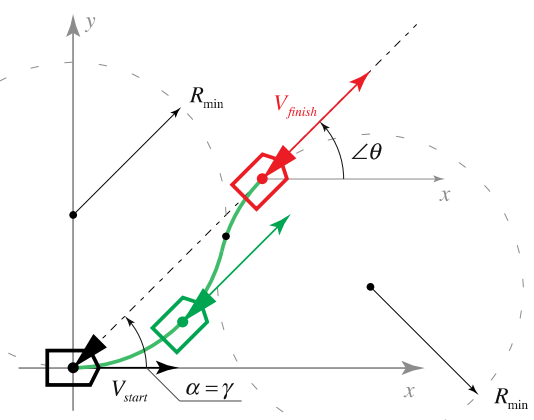

Fig. 1. Graphic representation of the trajectory for the first solution.

Fig. 1. Graphic representation of the trajectory for the first solution.

Figures 1 and 2 have following designations: $R_{\min }$ - the radius of the arc is the minimum radius which able to drive a car, $\alpha$ - the angle of orientation of the camera in the absolute coordinate system, measured from the axis of $x$. $\gamma$ angle of orientation of the camera, it is relative to the vector $V_{i}$ in the positive direction, $V_{\text {start }}, V_{\text {finish }}$ - orientation vectors of platform motion, in the initial and final points of the trajectory.

Consider these trajectories in more detail. Firstly, it should be noted that this trajectory is based on the consideration that the car-like platform there is some minimum radius of tuning by which it is able to move. The minimum radius (while maintaining the shape of the trajectory) provides the minimum distance to be held mobile robot first than leave the trajectory of the rectilinear motion in the direction of the initial sight chamber, secondly, each new 
part of trajectory must start from the point at which it is possible to hold one tangent for both new and old section, and the data points comes the steering wheel, so as to provide movement to the next path segment.

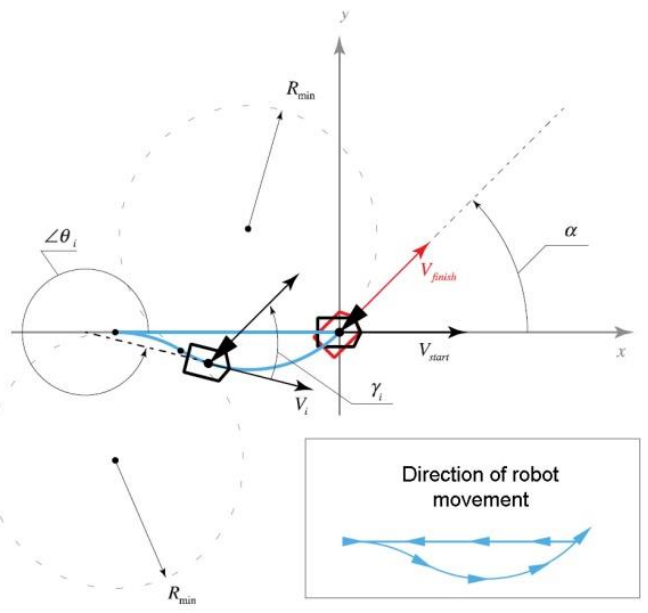

Fig. 2. Graphic representation of the trajectory for the second solution.

We find the equation for the construction of a first path consisting of two straight lines and two arcs, for convenience we introduce the new coordinate system with a reference point located at the starting point of the motion, the $x$-axis lies along the vector $V_{\text {start }}$ of the initial vehicle orientation axis $y$-normal to the $x$-axis as is shown on Fig. 3 .

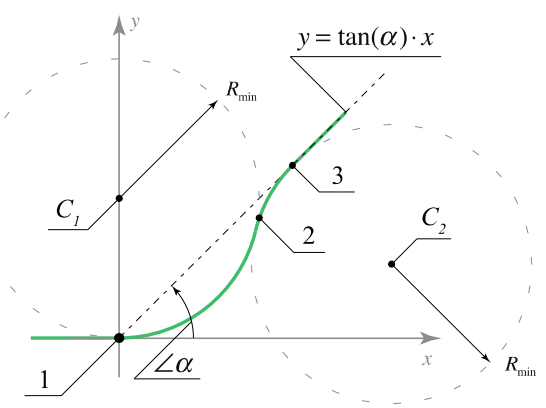

Fig. 3. Basic parameters necessary for the construction of the trajectory.

In Figure 3, the centers of the circles with the radius $R_{\min }$ marked as $C_{l}$ and $C_{2}$, and the numerals $1,2,3$ marked point of transition from one trajectory to another part.

The parametric equation of the first circle centered at $C_{l}$ :

$\left\{\begin{array}{l}x=R_{\text {min }} \cdot \cos (t) \\ y=R_{\text {min }} \cdot \sin (t)+R_{\text {min }}\end{array}, 0 \leq t \leq 2 \pi\right.$

This circle is constructed so that a point of contact with the point of the beginning of movement $(0,0)$, indicated as number 1 , and the point of contact with the second circle at the indicated as number 2 in Figure 3 , the coordinates of this point:

$0<\alpha \leq \frac{\pi}{2}:\left\{\begin{array}{l}x=\frac{-B+\sqrt{B^{2}-4 A \cdot C}}{4 A} \\ y=\frac{R_{\text {min }}+\frac{-b \sqrt{b^{2}-4 a \cdot c}}{2 a}}{2}\end{array}\right.$
$\frac{\pi}{2}<\alpha \leq \pi:\left\{\begin{array}{l}x=\frac{-B-\sqrt{B^{2}-4 A \cdot C}}{4 A} \\ y=\frac{R_{\min }+\frac{-b \sqrt{b^{2}-4 a \cdot c}}{2 a}}{2}\end{array}\right.$ 
where are A, B, C and a, b, c - the coefficients:

$$
\begin{aligned}
& a=\frac{1}{\tan ^{2}(\alpha)}+1 \\
& b=\frac{2 R_{\min }}{\sin (\alpha) \cdot \tan (\alpha)}-2 R_{\min } \\
& c=\left(\frac{1}{\sin ^{2}(\alpha)}-3\right)-R_{\min }^{2} \\
& A=\tan ^{2}(\alpha)+1 \\
& B=2 R_{\min } \cdot \tan (\alpha) \cdot\left(-\frac{1}{\cos (\alpha)}-1\right) \\
& C=R_{\min }^{2} \cdot\left(\frac{1}{\cos ^{2}(\alpha)}+\frac{2}{\cos (\alpha)}-3\right)
\end{aligned}
$$

Parametric equation of a second circle with center $\mathrm{C}_{2}$ :

$$
\left\{\begin{array}{l}
x=\frac{-B \pm \sqrt{B^{2}-4 A \cdot C}}{4 A}+R_{\text {min }} \cos (t) \\
y=\frac{R_{\min }+\frac{-b \sqrt{b^{2}-4 a \cdot c}}{2 a}}{2}+R_{\min } \sin (t)
\end{array}, 0 \leq t \leq 2 \pi\right.
$$

Now, as the first time we find a solution of the equation describing the trajectory for the second solution. In Figure 4 , the centers of the circles with the radius $R_{\min }$ marked as $C_{1}$ and $C_{2}$, and the numerals 1,2,3 marked point of transition from one trajectory to another part. The coordinates of the point marked by the number 1 :

$$
\left\{\begin{array}{l}
x=\frac{-2 R_{\min } \cdot \sin (\alpha) \sqrt{\left(4 \sin ^{2}(\alpha)-(8 \cos (\alpha)-8)\right)-R_{\min }^{2}}}{2} \\
y=0
\end{array}\right.
$$

The parametric equation of the first circle centered at $\mathrm{C}_{1}$ :

$$
\left\{\begin{array}{l}
x=\frac{-2 R_{\min } \cdot \sin (\alpha) \sqrt{\left(4 \sin ^{2}(\alpha)-(8 \cos (\alpha)-8)\right)-R_{\min }^{2}}}{2}+R_{\min } \cos (t)_{, 0 \leq t \leq 2 \pi} \\
y=-R_{\text {min }}+R_{\min } \sin (t)
\end{array}\right.
$$

This circle is constructed so that a point of contact at the indicated by number 1 , and the point of contact with the second circle at the indicated by number 2 in Figure 4 . The coordinates of point 2 :

$$
\left\{\begin{array}{l}
x=\frac{\frac{-2 R_{\min } \cdot \sin (\alpha) \sqrt{\left(4 \sin ^{2}(\alpha)-(8 \cos (\alpha)-8)\right)-R_{\min }^{2}}}{2}-R_{\min } \cos (t)}{2} \\
y=\frac{R_{\min } \cos (\alpha)-R_{\min }}{2}
\end{array}\right.
$$




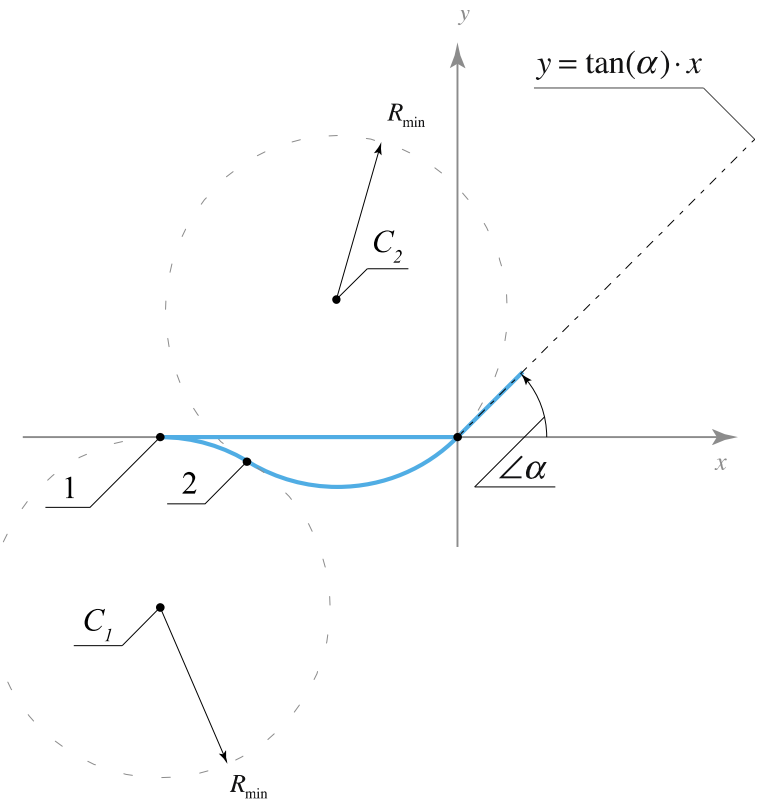

Fig. 4. The main parameters for the construction of the trajectory for the second solution.

The equation of the second circle with center $\mathrm{C}_{2}$ :

$\left\{\begin{array}{l}x=-R_{\min } \cdot \sin (\alpha)+R_{\text {min }} \cdot \cos (t) \\ y=R_{\text {min }} \cdot \cos (\alpha)+R_{\text {min }} \cdot \sin (t)\end{array}, 0 \leq t \leq 2 \pi\right.$

The second circle is the point of contact with the line $y=\tan (\alpha) \cdot x$ at the origin $[0,0]$.

At this stage, the trajectory obtained by moving along which the mobile platform is turning and the co-orientation vector accommodate platform motion vector rate mobile robot endpoint trajectory. Movement along a predetermined path runs along arcs centered at $C_{1}$ and $C_{2}$. The contact points $1,2,3$ there is a transition to the new phase of the trajectory.

\section{Preparation of the kinematic relations} Figure 5 .

It is necessary to find the kinematic relations for this type of platform, for this we consider view shown in

First, consider the representation of the platform (car-like platform) in the absolute coordinate system associated with the original position and orientation of the platform. Figure 5 has following designations: $\theta$ - the angle between the orientation vector $V$ is relative to the $o x$ axis in the positive direction, the angle $\phi$ - the angle of the steering wheel is relative to the axis of the connection of the front wheels and the rear, the point $P$ - point owned housing platform moving on a trajectory highlighted in blue. For the convenience of subsequent calculations arrange the camera at this point. It should be noted that the point $P$ may be selected arbitrarily, as when driving on an arc around its center, any point also moves relative to the center, but another radial direction.

A simplified kinematic model (without wheels slip on the surface) for mobile robots on the type of car-like taken from the following works $[7,8,9]$ :

$$
\left\{\begin{array}{l}
\dot{x}=v(t) \cdot \cos (\theta) \\
\dot{y}=v(t) \cdot \sin (\theta) \\
\dot{\theta}=\frac{v(t)}{L} \cdot \tan \phi \\
\phi=\arcsin \left(\frac{L}{R-\frac{l}{2}}\right)
\end{array}\right.
$$




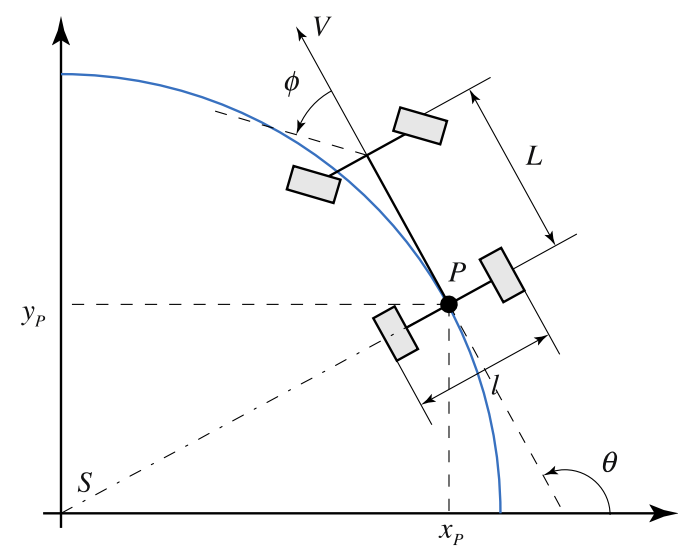

Fig. 5. Basic configuration car-like platform on the plane.

More model given in the book [8], we present it here, omitting the intermediate-transform education. The kinematic model of the car-like mobile robot can be reduced to the model of bike, with small values of the angle of the steering axle.

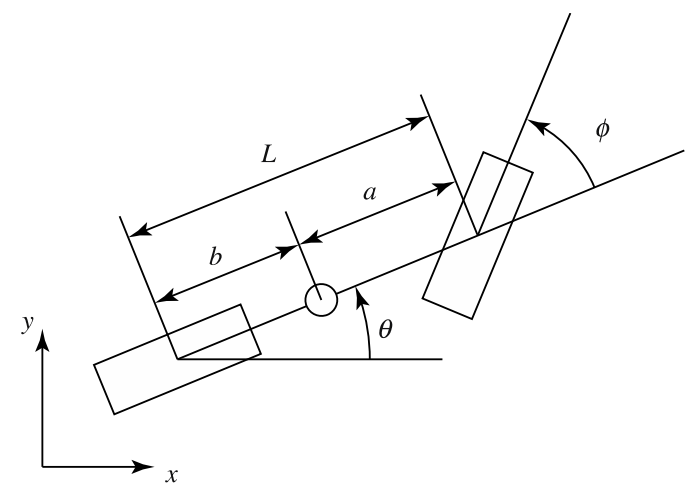

Fig. 6. Configuration of bike model which similar to model of car-like platform.

Figure 6 has following designations: $L$ - length of the bridge connecting the front and rear wheel, $b$ and $a$ - distance to the point where the center of mass is located.

If we neglect the wheel slip at the point of contact with the surface of the wheel, then there are non-holonomic constraints, defines a set of velocities:

$\dot{u} \cdot \sin (\theta)-\dot{w} \cdot \cos (t)=0$

where, $\dot{u}$ and $\dot{w}$ - the speed of the wheel in the coordinates $(u, w)$. Note coordinates $(x, y)$, as the position of the center of gravity $(C G)$ in the coordinate system $X, Y$. Note point $\left(x_{1}, y_{1}\right)$ - the coordinates of the drive wheel, $\left(x_{2}, y_{2}\right)-$ the coordinates of the steering wheel. Then

$\begin{array}{ll}x_{1}=x-b \cos (\theta), & x_{2}=x+a \cos (\theta), \\ y_{1}=y-b \sin (\theta), & y_{2}=y+a \sin (\theta), \\ \dot{x}_{1}=\dot{x}+b \dot{\theta} \sin (\theta), & \dot{x}_{2}=\dot{x}-a \dot{\theta} \sin (\theta), \\ \dot{y}_{1}=\dot{y}-b \dot{\theta} \cos (\theta), & \dot{y}_{2}=\dot{y}+a \dot{\theta} \cos (\theta) .\end{array}$

Then the equation of nonholonomic constraints for each wheel, taking into account (11) will be:

$$
\begin{aligned}
& \dot{x} \sin (\theta)-\dot{y} \cos (\theta)+b \dot{\theta}=0, \\
& \dot{x} \sin (\theta+\phi)-\dot{y} \cos (\theta+\phi)+a \dot{\theta} \cos (\theta)=0 .
\end{aligned}
$$

Using the coordinate system associated with the car, we introduce into consideration the $u$ axis lying along the length of the platform and the axis $w$ - axis normal to $u$. Rewrite considering this coordinate system a kinematic model of mobile robot: 
$\left\{\begin{array}{l}\dot{x}=v_{u} \cos (\theta)-v_{w} \sin (\theta) \\ \dot{y}=v_{u} \sin (\theta)+v_{w} \cos (\theta)\end{array}\right.$

where $v_{u}$ and $v_{w}$ projection velocity of the center of mass on the axis $u$ and $w$, respectively.

Substituting (13) into (12) and in view of the express:

$v_{w}=\dot{\theta} b$,

$\dot{\theta}=\frac{\tan \phi}{L} v_{u}$.

Then, the final equation of nonholonomic constraints will take the form:

$\dot{v}_{w}=\ddot{\theta} b$,

$\ddot{\theta}=\frac{\tan \phi}{L} \dot{v}_{u}+\frac{v_{u}}{L(\cos \phi)^{2}} \dot{\phi}$.

The angle $\phi$ depends on the construction of the steering axle, for systems with parallel turning wheels (non-Akermann steering):

$\phi=\arcsin \left(\frac{L}{R-\frac{l}{2}}\right)$

where $L$ - distance between front and rear axles, $R$ - the radius of the arc through which the robot will move at a given angle $\phi, l$ - distance between the wheels. The resulting mathematical description of kinematics of car-like platform will require when creating and testing the algorithm on the model of the robot in the environment of Matlab / Simulink.

\section{Development of algorithm of remote control movement of the mobile robot}

To accomplish the task - related commands motion control of the mobile robot with the vector-set orientation view camera installed on its body, developed the algorithm must imple-call of the automatic movement path thereby turn the mobile platform so as to ensure the further movement of the robot for a given operator rate . Upon rotation of the robot, the actuator is attached to the television camera which must be rotated in the opposite rotation direction in order to stabilize the orientation of the camera view yaw.

Movement along a predetermined path shown in Figures 1, 3 according to the following the algorithm:

1. In the event of the master joystick control commands movement of the mobile robot from the zero position, or when one of the keys also specify motion control commands, body of the main program, responsible for control of the mobile robot starts the execution of the program module.

2. We expect the desired angle of the course by the formula (3).

3. At point 1 control signal to the actuator turns the steering axle, the steering angle of the bridge is calculated by the formula (16).

4. After the steering axle, fed a control signal to drive the driving wheels, causing the robot to move from the first arc centered at $C_{1}$.

5. Reaching the point 2, the robot stops and turns the steering axle at an angle calculated by the formula (16).

6. After the steering axle, fed a control signal to drive the driving wheels, causing the robot to move at a second arc centered at $C_{2}$.

7. Reaching the point 3 , the robot stops and turns the steering axle at an angle $\phi=0$ that will provide linear motion for a given course.

\section{Mathematical modeling}

The experiment will be conducted for the general case in order to verify that the proposed algorithm. We give a block diagram of a system (see Fig. 7) on the basis of which to build a model in Simulink. 


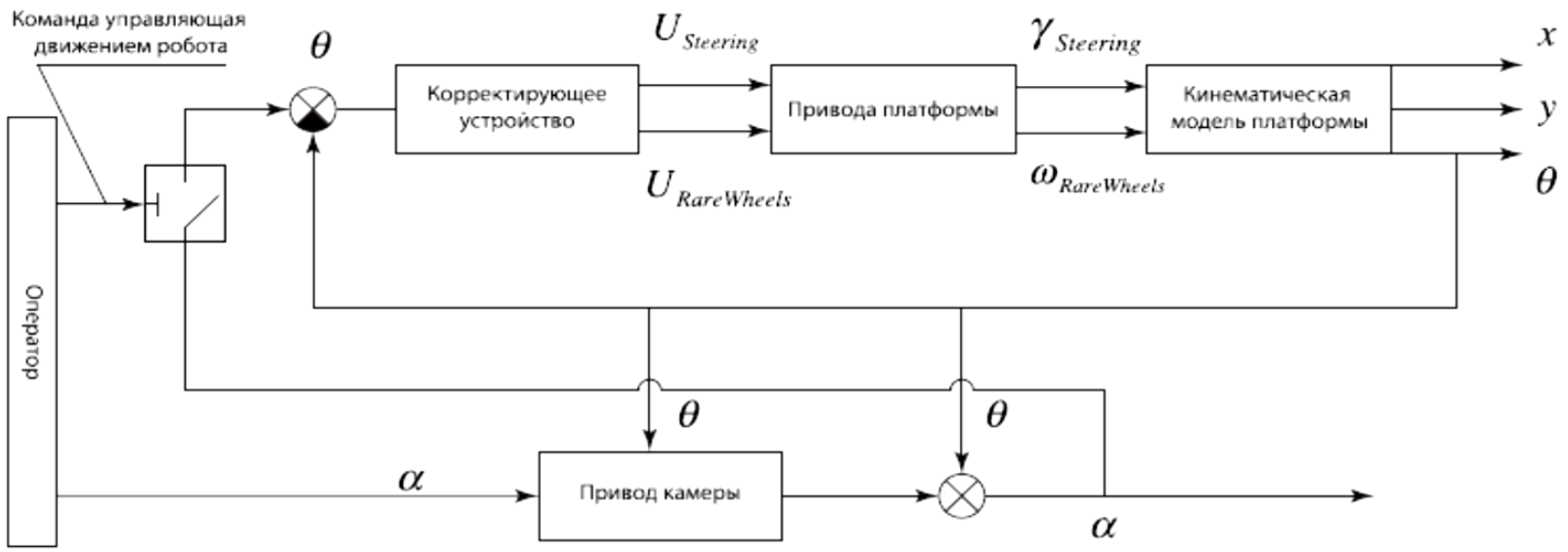

Fig. 7. A block diagram of a mobile robot vehicle type.

Figure 7 has following designations: $\alpha$ - angle of orientation of the camera in the absolute co-ordinates, $U_{\text {Rarewheels }} \mathrm{s}$ - input signals to the drive torque driving the rear pair of wheels, $U_{\text {Stering }}$ - the input signal to the actuator turns the steering axle, $\gamma_{\text {Stering }}$ - the angle of the steering axle, $\omega_{\text {Rarewhels }}-$ the angular velocity of rotation of the rear wheels. The output of the block with a kinematic model of the mobile platform already received, are: $\theta$ - the angle of orientation of the platform in the absolute coordinate system, $x, y$ - the coordinates of the robot in the absolute coordinate system.

The experiment consists in that the operator changes the orientation of the view camera at the starting point in the absolute coordinate system, and then shall submit the command "forward movement" for a mobile robot, wanting him to move forward in the direction of view camera. The platform should move along the path thereby aligning its vector orientation (course) with a given vector orientation of the camera, and continue to move forward towards the desired course.

We define a control signal which will turn on the camera orientation vector angle $\alpha=60^{\circ}$ an absolutecoordinate system. This trend will be further foreign exchange movement angle board-shape, which is only possible when $\theta=60^{\circ}$. The simulation results are presented in Figures 8, 9 .

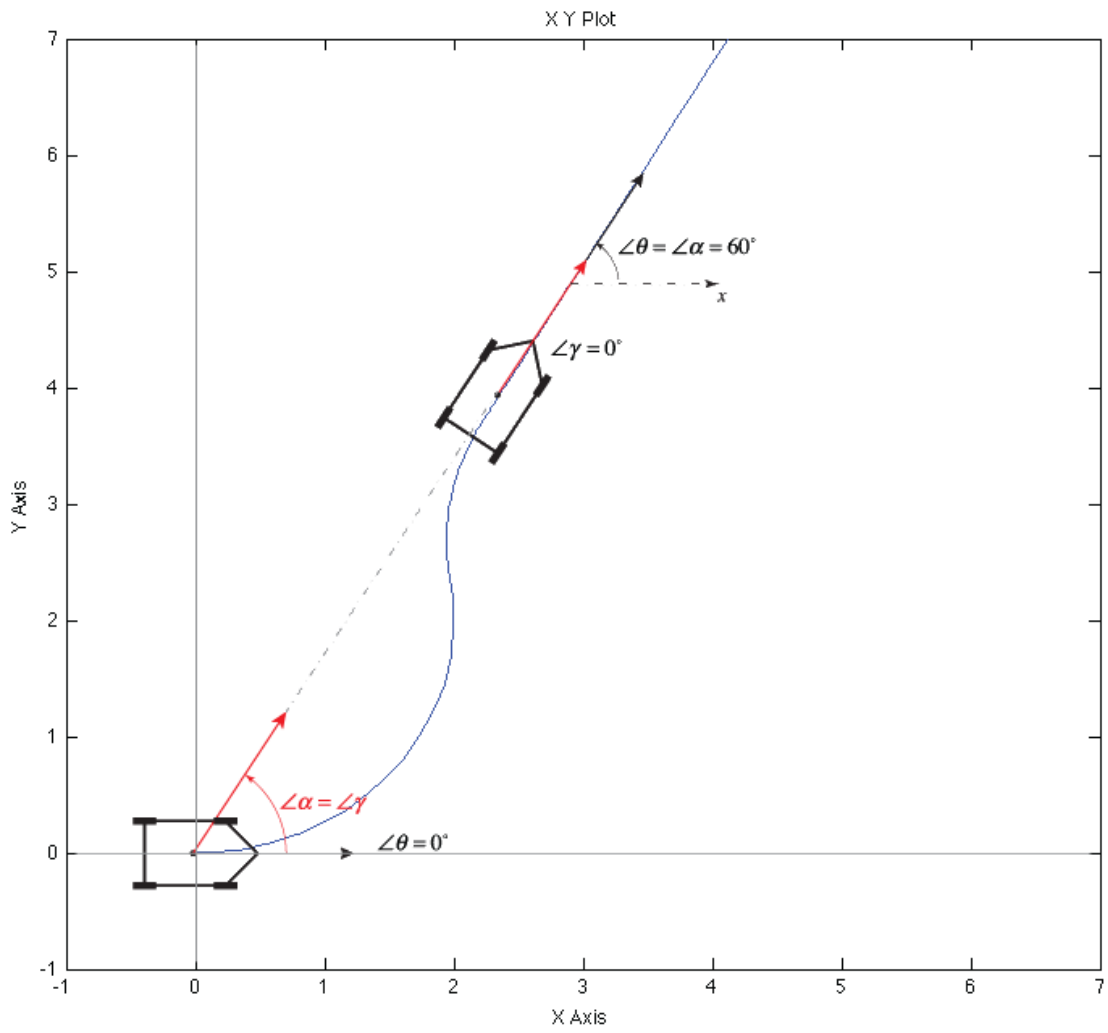

Fig. 8. Visualization of the trajectory traversed by a robot. 

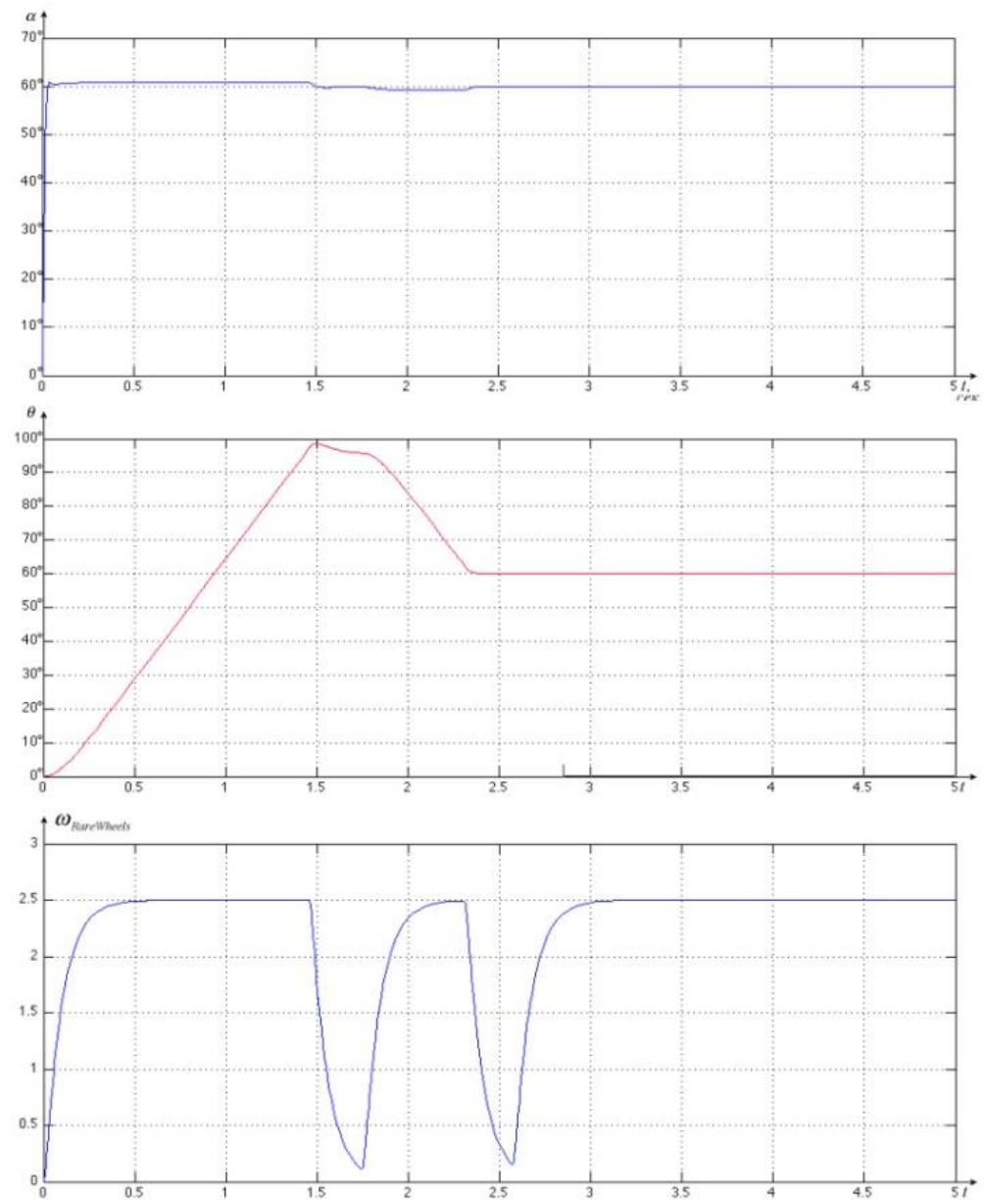

Fig. 9. Graph of the orientation angle of the camera, the angle of orientation of the platform and the wheel rotation angular velocity.

Consider Figure 9 in the interval from 0 seconds. to $2.4 \mathrm{sec}$. Platform performs motion along a curved path. At time points 1.4 and 2.4 seconds. the robot moves from one area to another path, for it is on, he slows down and rotates at a predetermined angle of steer - "moving forward", the platform begins to turn from $0^{\circ}$ to $60^{\circ}$, and approximately 3.4 sec., when the angle of view of the orientation of the camera becomes the angle of orientation of the platform $\alpha=\theta=60^{\circ}$, the robot starts to move straight ahead $60^{\circ}$. Upon executing the swing control system maintains a predetermined camera angle $\alpha=60^{\circ}$ to the drive chamber fulfills this angle $\Delta \gamma=-\Delta \theta$, i.e. rotated by an angle equal to the inverse change in the angle of orientation of the platform and eventually the rotation angle of the output shaft takes the initial, zero position in a coordinate system associated with the platform.

After model tests in Simulink has been tested for performance logic algorithm, which solves the above task - to bind commands movement of the mobile robot with the vector orientation view camera mounted on its casing, produce automatic rotation of the mobile platform to the specified operator of the corner of the course of motion of the platform and start traffic on given course.

\section{Conclusion}

The system offered in work allows considering automatically orientation of the television camera established on the body of the mobile robot during the formation of commands for robot motion control. For platform-type vehicle was designed algorithm allows to associate commands the robot with the vector orientation of the camera view. Due to the lack of full-scale model of this type of platform, the mathematical model of the system included in the control algorithm it was modeled and tested successfully on the performance in the work environment Matlab. As a result psychological load on the operator of the mobile robot considerably decreases, that raises its productivity.

\section{Acknowledgements}

The paper is supported by Russian Ministry of Education and Science (Agreement № 14.604.21.0054 от 30.06.2014, RFMEFI60414X0054). 


\section{References}

[1] Changhwan C., Yongchil S., Seungho J., Seungho K. Tele-Operated Fuel Handling Machine Manipulation Robot for the Nuclear Power Plants. Proc. CD-ROM of the 6th Asian Control Conf. Bali, Indonesia, 2006, pp. 983-987.

[2] Andreev V.P., Pryanichnikov V.E. Operation Environment of Mobile Robots with Supervision Control, Annals of DAAAM for 2011 \& Proceedings of the 22nd International DAAAM Symposium, Volume 22, No. 1, Vienna, Austria, 2011, pp. 0021-0022.

[3] D. Hwang, Teleoperation performance with a kinematically redundant slave robot, Ph.D. Dissertation, University of Washington, Department of Electrical Engineering.

[4] Cetin L., Baser O., Keskin O., Uyar E. Vision Based Control Algorithm for a Mobile Manipulator Annals of DAAAM for 2011 \& Proceedings of the 22nd International DAAAM Symposium, Volume 22, No. 1, Vienna, Austria, 2011, pp. 0449-0450.

[5] Lee D., Spong M.W. Passive bilateral control of teleoperators under constant time-delay, Proc.16 IFAC World Congress. Prague, Czech Rep., File 03009.pdf (CD-ROM), 2005, pp.1- 6.

[6] Tarca R., Pasc I., Tarca N., Popentiu-Vladicescu F. Remote robot control Via Internet Using Augmented Reality, Proc. of the 18th DAAAM Int. Symp. Intelligent Manufacturing \& Automation, Zadar, Croatia, 2007, pp. 739740 .

[7] Katsurin Alexey. System of Telecontrol by the Mobile Robot with Movable Camera // Applied Mechanics and Materials. Vol. 643. 2014. pp. 9-14.

[8] Vladimir Filaretov, Alexey Katsurin. Method of Semiautomatic Combined Control by Manipulator Using Mobile Telecamera. Proc. of 11th International Conference on Control, Automation and Systems (ICCAS 2011). KINTEX, Gyeonggi-do, Korea, 2011, pp. 649-654.

[9] Tomasz Kosicki and Trygve Thomessen. Cognitive Human-Machine Interface Applied in Remote Support for Industrial Robot Systems. International Journal of Advanced Robotic Systems, Vol. 10, 342, 2013. pp. 1-11.

[10] Soichiro Suzuki, Satoshi Hasegawa and Masayuki Okugawa. Remote control system of disaster response robot with passive sub-crawlers considering falling down avoidance. ROBOMECH Journal, Vol. 1, 20, 2014, pp. 1-12.

[11] Filaretov Vladimir, Katsurin Alexey. Method of Semiautomatic Position Control by Manipulator Using Telecamera Which Changes Its Orientation. Advanced Materials Research, Vol. 717, 2013, pp. 573-578.

[12] Angeles, J. Fundamentals of Robotic Mechanical Systems: Theory, Methods, and Algorithms, 3rd Edition, SpringerVerlag, ISBN-10: 0387945407, New York, 2006.

[13] Bruno Siciliano, Handbook of Robotics / Bruno Siciliano, Ousamma Khatib. - Berlin, Springer, 2007. - 1591 c.

[14] Eric N Moret, Dynamic Modeling and Control of a Car-Like Robot / Eric N Moret. - Blacksburg, Virgin, 2003. $78 \mathrm{c}$.

Patricia Mellodge, Feedback Control for a Path Following Robotic Car / Patricia Mellodge. - Blacksburg, Virgin, 2002. $-117 \mathrm{c}$. 